\title{
The Case Manager: Driving Medical Reasoning in a Distributed Environment for Home Patient Monitoring
}

\author{
Francesca POLCE ${ }^{\mathrm{a}, 1}$, Giordano LANZOLA ${ }^{\mathrm{a}}$, Matteo GABETTA ${ }^{\mathrm{b}}$, Enea \\ PARIMBELLI $^{\mathrm{a}}$, Szymon WILK ${ }^{\mathrm{c}}$, David GLASSPOOL ${ }^{\mathrm{d}}$, Roy LEIZER ${ }^{\mathrm{e}}$, Alexandra \\ KOGAN $^{\mathrm{e}}$ and Silvana QUAGLINI ${ }^{\mathrm{a}}$ \\ a Dept. of Electrical, Computer and Biomedical Eng., University of Pavia, Pavia, Italy \\ ${ }^{\mathrm{b}}$ Biomeris S.r.l., Pavia, Italy \\ ${ }^{\mathrm{c}}$ Inst. of Computing Science, Poznan University of Technology, Poznan, Poland \\ ${ }^{\mathrm{d}}$ Deontics Ltd., London, United Kingdom \\ ${ }^{\mathrm{e}}$ Dept. of Information Systems, University of Haifa, Haifa, Israel
}

\begin{abstract}
The CAPABLE project has been funded by the EU Horizon 2020 Programme over the years 2020-24 to support home care. A system is being designed and implemented supporting remote monitoring and virtual coaching for cancer patients. The system is based on a distributed modular architecture involving many components encapsulating various knowledge. The Case Manager has been designed as a separate component with the aim of coordinating the problem solving strategies. A first version of the Case Manager has been released and used by the components in a prototypical scenario shown at the first project review.
\end{abstract}

Keywords. Telemedicine, remote monitoring, shared problem solving, node coordination

\section{Introduction}

The evolution of demographic dynamics and the consequent changes in the health needs of the population call for a structural and organizational redesign of the health services. Telemedicine systems are already able to support the transition of health care from hospitals to the assisted patient homes [1]. Moreover, the global health emergency that we have been experiencing through the COVID-19 epidemic also shows the potential of introducing Telemedicine in everyday life [2] as a means of remotely supporting the interaction between patients and clinicians. This innovative digital care model promotes patient self-management, reducing hospitalization episodes and saving related costs. Thanks to the wide adoption of smartphones, tablets and smartwatches, patients have tools that allow the collection and transmission of clinical data captured through sensors along with Patient Reported Outcomes (PROs) and Patient Reported Experience (PREs) without waiting for scheduled visits. This approach can potentially collect an enormous amount of information that should be analyzed and interpreted automatically exploiting

1 Corresponding Author, Francesca Polce, Department of Electrical, Computer and Biomedical Engineering, University of Pavia, Via Ferrata 5, Pavia, Italy; E-mail: francesca.polce01@universitadipavia.it. 
Artificial Intelligence methodologies and Big Data processing techniques that reached a high maturity level in the last few years [3].

With this picture in mind, the CAPABLE (CAncer PAtients Better Life Experience) project was started, funded by the Horizon 2020 Programme for Research and Innovation over the years 2020-24. CAPABLE tries to combine the most advanced technologies mentioned above to develop a home monitoring system promptly informing clinicians about any criticality. It also provides virtual coaching for patients, increasing their adherence to the treatment and improving their quality of life.

\section{Methods}

CAPABLE was conceived as a modular distributed system, as shown in Figure 1 illustrating the most important modules that make up its architecture. Through the mobile app, the system acquires data either directly from the patient or his caregiver, or from environmental or wearable sensors. Those data are merged with clinical information extracted from the Electronic Health Record made available by the hospital where the patient is being treated. The system also involves a variety of heterogeneous Knowledge Sources (KSs) addressing the management of cancer patients. KSs continuously process the patient data stored on the Data Platform (DP) incrementing them with new information in the form of forecasts, warnings, or partial interpretations (e.g. qualitative data abstractions). KSs include modules for rendering computer-interpretable clinical practice guidelines, predictive models addressing cancer evolution, the Virtual Coach providing recommendations to the patient, the Multimorbidity Controller detecting and resolving conflicts due to multiple diseases coexistence on the same patient and the Knowledge-Data Ontology Mapper (KDOM) responsible for carrying out clinical abstractions. Predictive models are initialized by leveraging training data relating to previous patients made available by the CAPABLE's medical partners.

KSs are developed as pluggable components that can be added or removed without disrupting the system overall reasoning ability. To achieve this goal each component must be fully decoupled from the others. We adopted a Blackboard model, which is one of the most versatile solutions found in the literature [4]. Therefore, KSs only interact through the DP, seen as a shared blackboard where information concerning the patient is published by a component and read by any other interested one. To prevent components from continuously scanning the blackboard, CAPABLE provides an additional component called Case Manager (CM), specifically designed to coordinate the KSs and activate the right ones only when needed. This recalls similar attempts already available in the literature [5]. The CM guides the reasoning process through an Event triggering strategy, where Events are combinations of facts that represent the main updates regarding the patient's clinical status. The $\mathrm{CM}$ monitors the DP to detect the occurrence of such Events, so that the interested KS can be promptly notified, resuming a reasoning process that has previously been "paused" waiting for the arrival of new data. Thus, CM is the only component allowed to send messages directly to any other one, promoting a clear design and a uniform communication protocol.

The interaction for exchanging health data between components is facilitated, at the semantic level, by the adoption of the emerging FHIR (Fast Healthcare Interoperability Resources) standard Release 4 developed by HL7 (Health Level Seven). To this aim, we mapped all data used by CAPABLE into the corresponding FHIR Resources (i.e. Patient, Observation, MedicationRequest, Communication, Goal, etc.). 


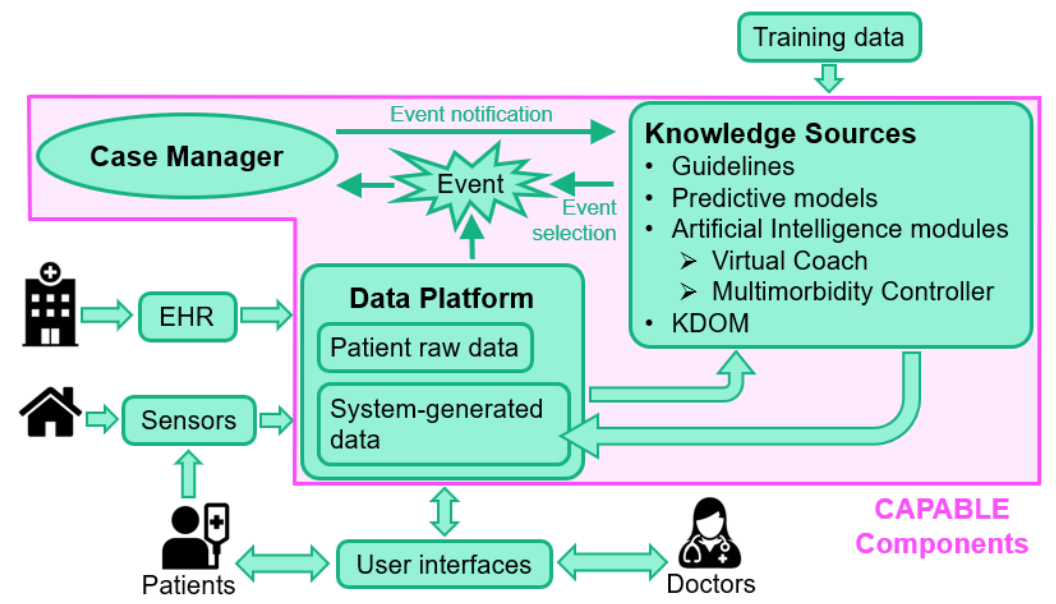

Figure 1. The high-level architecture adopted by CAPABLE.

The CM sits between the DP on one side and the rest of the CAPABLE components on the other, as shown in Figure 2 illustrating the communication model. First, the components inform the CM about their Events of interest creating a Rule (links 1.1/1.2 in Figure 2) for each Event to be monitored. Rules contain all the information required by the $\mathrm{CM}$ to generate the corresponding Event and are made up combining two main parts: the data filters and logic. The filters contain the FHIR criteria used by the CM to retrieve data useful for generating Events from the DP (link 2.1 in Figure 2), while the logic performs additional controls on the fetched data checking for the effective generation of the Event. In addition, each time new data are entered in the DP, the CM is also notified (link 2.2 in Figure 2). Finally, the CM implements a notification mechanism to inform the components about the Events occurrence (link 3.1 in Figure 2).

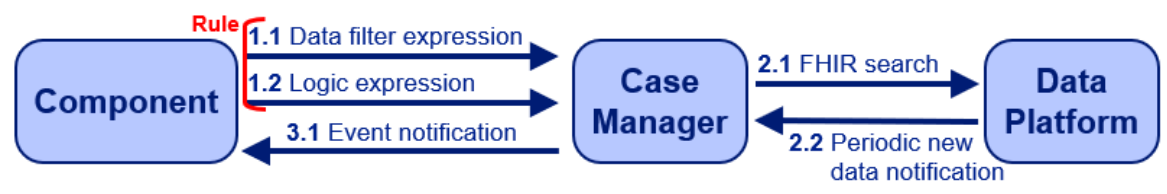

Figure 2. Communications diagram illustrating the CM operating model.

\section{Results}

We defined a syntax based on JSON (JavaScript Object Notation), shared among all the CAPABLE components, for defining Rules. As shown in Figure 3 (A) a rule includes: a configuration part with the unique name of the Rule, the frequency for re-evaluating it and other optional features; the search filters exploiting the parameters that FHIR makes available to fetch Resources from the DP; a logic expression containing JavaScript code injected and run directly on the CM. This code encapsulates checks on the Resources to finely control Event activation (e.g. digging deeper into the Resource model or making comparisons between them). While checking Rules, the CM stores any partial evaluation in a cache to avoid continually querying the DP. As shown in Figure 3 (B), each Rule is associated with a tree that develops along different lines, one for each specific patient, 
in which fetched Resources are incrementally stored. The tree lines are populated when a Rule is initially defined by issuing the FHIR search queries on the DP. Then, every time the DP announces to the CM the addition of a new Resource, the latter is tested locally by the CM to verify if it satisfies the filters of that Rule. If so, the Resource will be stored in the corresponding tree line. When the CM finds that the Resources retrieved satisfy all the conditions referenced by the Rule (both search filters and logic), it generates the Event and sends it to the interested component, together with all the Resources. The involved tree line is then emptied and rebuilt from scratch.

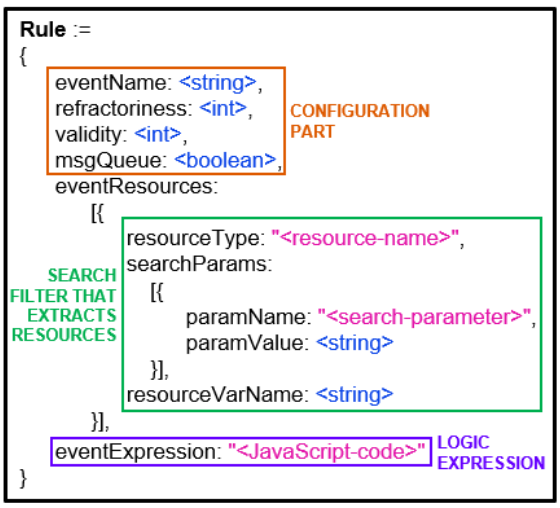

(A)

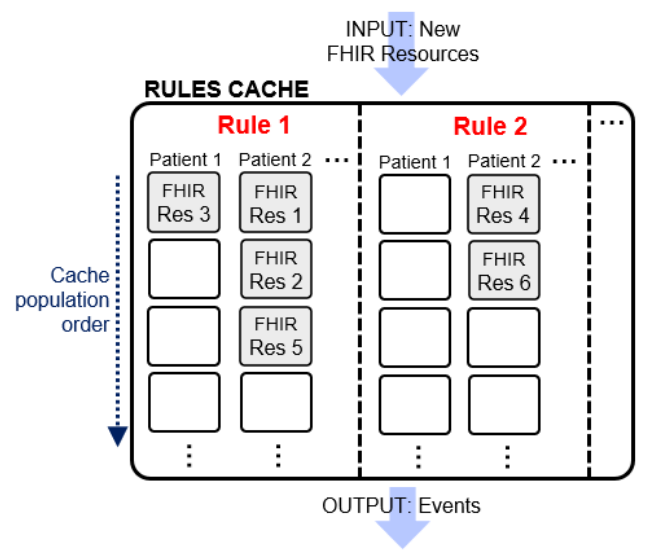

(B)

Figure 3. (A) The JSON syntax for defining Rules; (B) The CM cache where FHIR Resources partially satisfying the Rules are stored.

To simplify the CAPABLE system implementation, we started from a clinical usecase scenario addressing the ESMO Diarrhea guideline [6], which is used for treating this side effect of cancer immunotherapy. The sequence diagram in Figure 4 schematizes a small part of it, highlighting how the $\mathrm{CM}$ coordinates the different components through Events. In this case, a patient experiences a grade 2 diarrhea episode according to the CTCAE (Common Terminology Criteria for Adverse Events) and self-reports it to the system via the mobile app. This symptom is stored within the DP as a FHIR Observation Resource. The CM is then notified about the arrival of new relevant data for the Virtual Coach (i.e. satisfying one of its Rules). Thus, the latter receives the Event regarding a new symptom and asks the KDOM to check if diarrhea can be classified as complicated. This is an abstraction useful for choosing the right recommendation for the patient. The message exchanged between the two components is stored in the DP as a Communication Resource instance causing the activation of a new Event for the KDOM, and so on.

\section{Discussion and Conclusions}

The coordination among the components in CAPABLE is essential because elaborating a diagnosis or suggesting a treatment is a complex activity that requires knowledge spread over the various KSs. The CM dynamically invokes components sending Events that are generated through the Rules provided by them and fitting their needs. 


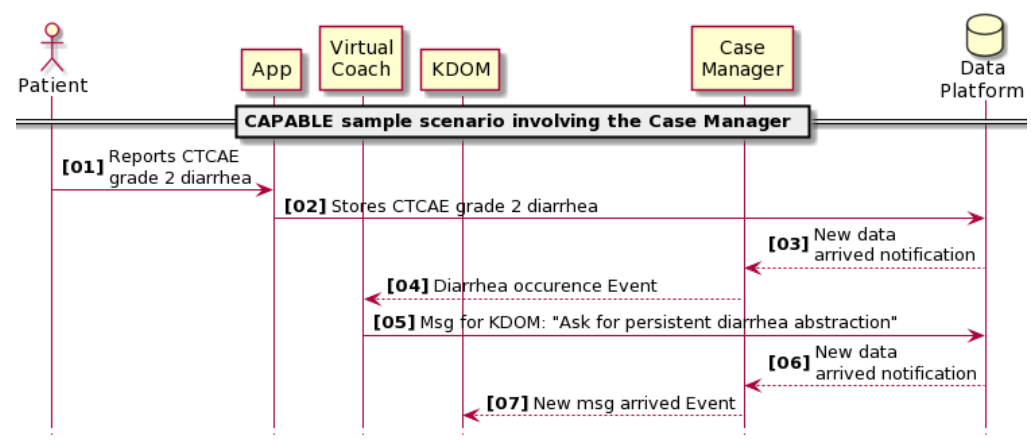

Figure 4. Sequence diagram describing the interactions among some CAPABLE components and the CM in the clinical scenario developed to validate the system behavior.

A first implementation of the $\mathrm{CM}$ was released as a shared Virtual Machine instance for use by the other components. As a preliminary evaluation, we used Postman (www.postman.com) as a debugging tool to ensure the correct functioning of the CM and its performance. First, we designed several test scenarios, such as the one described above, to reflect the CM normal conditions in the context of CAPABLE once the system goes into production. The simulations were carried out in an automated way to test the core system functionality, the interactions between the $\mathrm{CM}$ and the other components and the response times. We also designed a series of stress tests aimed at determining the CM stability in heavy load conditions, not expected during normal system operation. All the tests carried out were successful and no critical issues emerged. Events were correctly generated by the $\mathrm{CM}$ and notified to the right component even when many FHIR Resources to be processed were entered in a short period of time. The CM was then integrated into the CAPABLE system and used for the first annual review of the project.

\section{Acknowledgements}

The work described in this paper has been funded through the H2020 EU grant n. 875052 to the Capable project.

\section{References}

[1] Lanzola G, Losiouk E, Del Favero S, Facchinetti A, Galderisi A, Quaglini S, Magni L, Cobelli C. Remote blood glucose monitoring in mHealth scenarios: a review. Sensors. 2016 Dec 1;16(12):2-16.

[2] Mann DM, Chen J, Chunara R, Testa PA, Nov O. COVID-19 transforms health care through telemedicine: Evidence from the field. Journal of the American Medical Informatics Association. 2020 Jul 1;27(7):1132-1135.

[3] Mehta N, Pandit A, Shukla S. Transforming healthcare with big data analytics and artificial intelligence: A systematic mapping study. Journal of Biomedical Informatics. 2019 Dec;100.

[4] Mesa I, Sanchez E, Diaz J, Toro A, Artetxe A, Grana M, Guijarro F, Martínez C, Jiménez JM, Alarcon JA, De Mauro A. GoCardio - A novel approach for mobility in cardiac monitoring. InImpact: The Journal of Innovation Impact. 2016 Oct;6(1):110-120.

[5] Falcionelli N, Sernani P, Brugués A, Mekuria DN, Calvaresi D, Schumacher M, Dragoni AF, Bromuri S. Indexing the Event Calculus: Towards practical human-readable Personal Health Systems. Artificial Intelligence in Medicine. 2019;96:154-166.

[6] Bossi P, Antonuzzo A, Cherny NI, Rosengarten O, Pernot S, Trippa F, Schuler U. Diarrhoea in adult cancer patients: ESMO Clinical Practice Guidelines. Annals of Oncology. 2018 Oct 1;29(4):126-142. 